\title{
Stability Analysis for Unknown Delayed Systems Controlled by Model-free Control
}

\author{
Maxime Doublet ${ }^{1}$, Cédric Join ${ }^{1,2,3}$, Frederic Hamelin ${ }^{1}$ \\ ${ }^{1}$ Université de Lorraine, CRAN, UMR 7039 Vandouvre-lès-Nancy 54500, France \\ 2 ALIEN (ALgèbre pour Identification et Estimation Numériques), 24-30 rue Lionnois, BP 60120, 54003 Nancy, France \\ ${ }^{3}$ Projet NON-A, INRIA Lille-Nord-Europe, France
}

\begin{abstract}
Unknown delayed systems are present in many domains and control this kind of systems remains a difficult problem. Indeed, we can find theses systems in physics, chemistry, aeronautics, etc. The main problem is that the delay is unknown, and so the model is not exactly well-known. By the way, the use of model-free control (MFC) remains a suitable solution to tackle this problem. This paper deals with a stability analysis to show the influence of a parameter on the stability of the systems regarding to the delay.
\end{abstract}

Keywords-Model-free control; stability analysis; unknown delayed system.

\section{INTRODUCTION}

In industry, we can find a lot of delayed systems in different domains. Sometimes the delay is well-known, but in many cases it is unknown. However classical methods which can be applyied when the delay is well-known are not suitable in the unknown delayed system context. For example the Smith predictor [10], [13], is a usual controller but it is adjusted with respect to the known delay. Obviously if the delay is unknown it is impossible to apply this method.

From several years an interesting approach was proposed by M.Fliess and C.Join [1] : model-free control (MFC). With this method, many kind of systems can be controlled [3] [8]. But, control unknown delayed systems believes a difficult problem. Indeed, in an unknown delayed system context difficulties arise quickly (stability, loss of velocity, etc.). In order to tackle these problems and improve the performances, a model-free design was proposed [4]. Moreover, an other advantage of this method against the classics, as model predictive control [11], is the easier implementation [2].

In this paper, we propose an frequential analysis of the stability of unknown delayed system. Moreover, we show the importance of parameter tuning for model-free control.

According to different general examples, we explain the method step by step. A frequential analysis is made to prove the stability of the system.

The paper is organized as follows, we firstly present the aim of this paper. The third section presents the general method to analyse the stability, i.e., the mean to obtain the closed-loop and the open-loop transfer functions as well as the frequential analysis. In the next section, the method described in the third section is applied on two systems. Finally a conclusion is given with perspectives.

\section{Problem STATEMENT}

We consider in this contribution unknown delayed systems as follows:

$$
\frac{Y(s)}{U(s)}=e^{-\tau s} G(s)
$$

where:

- $Y(s)$ is the output of the system,

- $U(s)$ is the input of the system,

- $\tau$ is the delay assumed to be unknown,

- $G(s)$ is a stable transfer function.

These systems are controlled by the model-free control approach. The aim here is to find a method to analyse the stability of the global system, i.e. the system and the controller. With MFC, we can act on three parameters: the tuning of the controller applied on the error tracking, the degree of derivation of the ultra-local model and the parameter which appears in the ultra-local model as explained later. We will focus on this last one and we will show that its tuning is very important because it affects directly the stability of the closed loop system.

\section{GENERAL METHOD}

In this section, the general method to analyse the stability of a system controlled by MFC is detailled. In a first time, we explain how to obtain the different transfer functions, between $Y_{\text {ref }}(s)$ the reference signal and $Y(s)$ the output of the system, in closed and open loop. Then, by using these equations, the general method to analyse the stability is given thanks to the Nyquist stability criterion.

\section{A. Obtain the open and closed-loop transfer functions}

In this subsection, we will see how to get the different transfer functions.

We consider the general system (1). As said before, this system is controlled by the MFC. With this approach we search the input/output rule of the system as:

$$
\dot{y}(t)=F(t)+\alpha u(t)
$$

where :

- $\dot{y}(t)$ is the first time derivative of the output,

- $F(t)$ groups all the unknown signals : disturbances, the imperfections of the model, etc. In implementation, it is estimated at each sample time, 
- $\alpha$ is, at first, a constant and it can be viewed as a scaling between the input and the derivative of the output,

- $u(t)$ is the input.

Assuming the estimation of $F(t)$ the control law is designed as:

$$
u(t)=\frac{1}{\alpha}\left(-F(t)+\dot{y}_{r e f}(t)\right)-C(e(t))
$$

Where $C(e(t))$ is generally a $P I D$ controller.

Using equations (1), (2) and (3) we will see how to obtain the closed-loop transfer function between $Y(s)$, the output, and $Y_{\text {ref }}(s)$, the reference.

Firstly, two remarks have to be made:

remark 1: A difference between $\dot{y}(t)$ and $\dot{y}_{\text {ref }}(t)$ is made. Indeed, $y_{\text {ref }}$ is a signal which is exactly known, so we can calculate its time derivative directly. It becomes in the Laplace domain: $y_{r e f}(t) \stackrel{L}{\rightarrow} s Y_{\text {ref }}(s)$. But, $y(t)$ is a measured signal, so we need to approximate its time derivative using a classic finite difference [12]. It gives:

$$
\hat{\dot{y}}(t) \stackrel{L}{\rightarrow} Y(s)\left(\frac{1-e^{-T_{e} s}}{T_{e}}\right)
$$

remark 2: (2) is the continous time model, but, in implementation, this model changes a bit. Indeed, to do not have algebraic loops during the resolution, we use the "previous" $u(t)$, so (2) becomes: $\dot{y}(t)=F(t)+\alpha u\left(t-T_{e}\right)$ and in the Laplace domain using (4) also:

$$
Y(s)\left(\frac{1-e^{-T_{e} s}}{T_{e}}\right)=F(s)+\alpha U(s) e^{-T_{e} s}
$$

Now, using (3), (4) and (5), we obtain in the Laplace domain:

$$
\begin{aligned}
U(s)=-\frac{Y(s)}{\alpha} & \left(\frac{1-e^{-s T_{e}}}{T_{e}}\right) \\
& +U(s) e^{-T_{e} s}+\frac{s Y_{r e f}(s)}{\alpha}-C(s) E(s)
\end{aligned}
$$

Finally, by using (1) to replace $U(s)$ in (6), we obtain the closed-loop transfer function $F_{C L}(s)$ between $Y(s)$ and $Y_{\text {ref }}(s)$ :

$$
\frac{Y(s)}{Y_{r e f}(s)}=\frac{T_{e}(s+\alpha C(s))}{G(s)^{-1} T_{e} \alpha e^{\tau s}\left(1-e^{-T_{e} s}\right)+A(s)}
$$

with $A(s)=\left(1-e^{-T_{e} s}+T_{e} \alpha C(s)\right)$.

Finally, it is well-known that for a unit gain return we have:

$$
F_{C L}(s)=\frac{N(s)}{D(s)}=\frac{F_{O L}(s)}{1+F_{O L}(s)}
$$

And, the open-loop transfer function is as follows:

$$
F_{O L}(s)=\frac{N(s)}{D(s)-N(s)}
$$

Now that we have got the open-loop transfer function, a frequential analysis using the Nyquist stability criterion can be made. It is the subject of the following subesction.

\section{B. Nyquist stability criterion}

In this section, based on the Nyquist stability criterion, the general method to analyse the stability of an unknown delayed system controlled by MFC is presented. The criterion is applied using the open-loop transfer function found previously for the considered example. The same analyse can be made with any other system. We decided to use the Nyquist stability criterion because another method as the Routh criterion can not be applied because of the delay.

According to the general open-loop transfer function obtained by (9), a frequential analysis can be proposed to study the stability of the system. To show the influence of the delay $\tau$ and the parameter $\alpha$ on the stability, the Nyquist diagram of the $F_{O L}$ is plotted.

To apply the Nyquist criterion, we need to find if the $F_{O L}$ has got unstable zeros and poles.

For the zeros, it is generally trivial. Concerning the poles, in our case, because of the complexity of the denominator we will use this following method. We assume that $s=x+j y$ and we need to find $x$ and $y$ whose make the denominator equal to zero, i.e. we search the tuple $(x, y)$ which verifies:

$$
|\operatorname{Im}(\operatorname{den}(x, y))|+|\operatorname{Re}(\operatorname{den}(x, y))|<\epsilon
$$

Usually, we want to find the tuple $(x, y)$ which make $|\operatorname{Im}(\operatorname{den}(x, y))|+|\operatorname{Re}(\operatorname{den}(x, y))|=0$, but because of the discretization, we use (10). Then, we discretize the space on $x$ and $y$ to solve numerically the problem because the equations are too complex and it is generally difficult to obtain an analytic solution. We plot the logarithm of the sum of the absolute value of the imaginary and real parts to find the number of unstable poles of the transfer function. We use the logarithm because it allows to highlight the zones where the function is minimal.

Once we have the number of unstable poles and zeros, applying the generalized Nyquist stability criterion we find that the number of encirclements of the critical point $(-1,0)$ in the complex plane has to be equal to the difference between the number of unstable poles and zeros. Indeed, the Nyquist stability criterion specifies that : $N=Z-P$ where, $N$ is the number of encirclements (clock-wise) of the point $(-1,0), P$ is the number of unstable poles and $Z$ is the number of unstable zeros [6], [7].

In the next section, the proposed method is applied on a first order system and a second order system.

\section{Application}

\section{A. First order system}

In this subsection, we apply the proposed method on this academical example:

$$
\frac{Y(s)}{U(s)}=\frac{e^{-\tau s}}{s+3}
$$

which is a first stable unknwon delayed system. In a first time we have to obtain the closed-loop and the open-loop transfer function. The controller considered here is a simple 
proportional corrector, i.e. $C(s)=K_{p}=0.05$. The closedloop transfer function is:

$$
\frac{Y(s)}{Y_{\text {ref }}(s)}=\frac{T_{e}\left(s+\alpha K_{p}\right)}{e^{\tau s} T_{e} \alpha(s+3)\left(1-e^{-T_{e} s}\right)+A(s)}
$$

Using (9), the open-loop transfer function is defined such as:

$$
F_{O L}(s)=\frac{T_{s}\left(s+\alpha K_{p}\right)}{e^{\tau s} T_{e} \alpha(s+3)\left(1-e^{-T_{e} s}\right)+A(s)-T_{e}\left(s+\alpha K_{p}\right)}
$$

With $A(s)=\left(1-e^{-T_{e} s}+T_{e} \alpha K_{p}\right)$.

Now, the aim is to obtain the number of unstable poles and zeros. For the zeros, it is trivial, we obtain:

$$
z_{1^{s t}}=-\alpha K_{p}
$$

For the poles, we separate the imaginary and the real part as specified in the previous section. For the imaginary part we have:

$$
\begin{gathered}
\operatorname{Im}(\operatorname{den}(x, y))=T_{e} \alpha\left(-3 e^{x\left(\tau-T_{e}\right)} \cos (\tau y) \sin \left(-T_{e} y\right)\right. \\
-x e^{x\left(\tau-T_{e}\right)} \cos (\tau y) \sin \left(-T_{e} y\right)-y e^{x\left(\tau-T_{e}\right)} \cos (\tau y) \cos \left(-T_{e} y\right) \\
-3 e^{x\left(\tau-T_{e}\right)} \sin (\tau y) \cos \left(-T_{e} y\right)-x e^{x\left(\tau-T_{e}\right)} \sin (\tau y) \cos \left(-T_{e} y\right) \\
\left.+y e^{\tau x} \cos (\tau y)+3 e^{\tau x} \sin (\tau y)+x e^{\tau x} \sin (\tau y)\right) \\
-e^{-T_{e} x} \sin \left(-T_{e} y\right)-T_{e} y
\end{gathered}
$$

And for the real part:

$$
\begin{array}{r}
\operatorname{Re}(\operatorname{den}(x, y))=T_{e} \alpha\left(e^{\tau x} \cos (\tau y)(3+x)\right. \\
-y e^{\tau x} \sin (\tau y)-3 e^{x\left(\tau-T_{e}\right)} \cos (\tau y) \cos \left(-T_{e} y\right) \\
-x e^{x\left(\tau-T_{e}\right)} \cos (\tau y) \cos \left(-T_{e} y\right)+y e^{x\left(\tau-T_{e}\right)} \cos (\tau y) \sin \left(-T_{e} y\right) \\
+y e^{x\left(\tau-T_{e}\right)} \sin (\tau y) \sin \left(-T_{e} y\right)+3 e^{x\left(\tau-T_{e}\right)} \sin (\tau y) \sin \left(-T_{e} y\right) \\
\left.+x e^{x\left(\tau-T_{e}\right)} \sin (\tau y) \sin \left(-T_{e} y\right)+y e^{x\left(\tau-T_{e}\right)} \sin (\tau y) \sin \left(-T_{e} y\right)\right) \\
-e^{-T_{e} x} \cos \left(-T_{e} y\right)-T_{e} y+1
\end{array}
$$

Using equations (15) and (16), we plot the logarithm of the sum of the absolute value of the imaginary and real part of the denominator as shown on figure 1 .

We have to focus on the unstable poles. It is why the second plot focuses on the zone where $x>0$. So, the $F_{O L}$ of the system does not have any zeros or poles unstable when $\alpha=3$ and for a delay $\tau=5 T_{e}$ where $T_{e}=0.1[\mathrm{~s}]$ as we can see on figure 1 .

All the tests have been made with different values for the parameters $\alpha$ and $\tau$ and the result is the same every time: the $F_{O L}$ does not have any unstables poles and zeros. So, applying the generalized Nyquist stability criterion we find that the Nyquist diagram has to make zero encirclement around the critical point.

Now, we will analyse the stability of the system for different values for $\alpha$ and $\tau$. All the different results are shown in the following array:
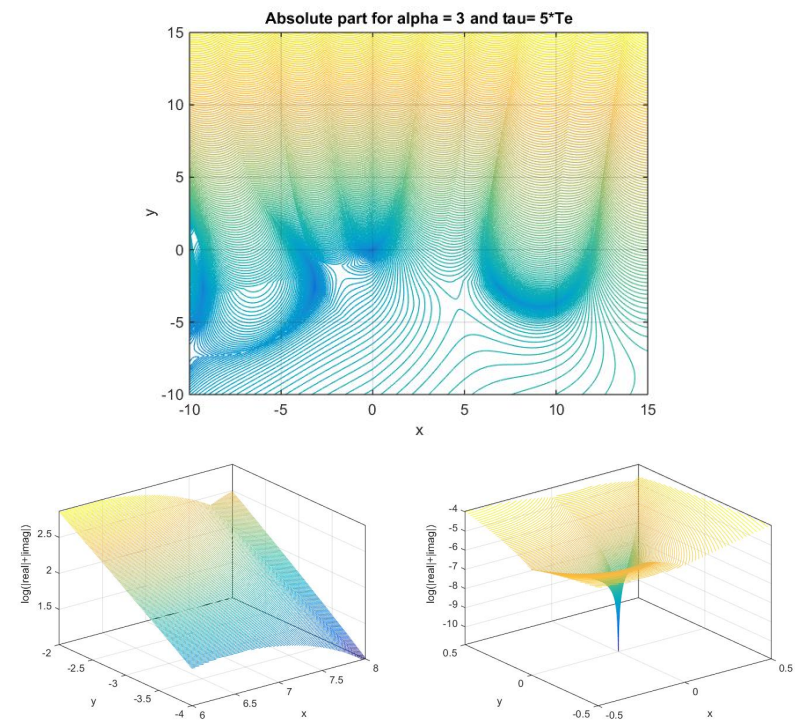

Figure 1. Logarithm of the sum of the absolute part of the real and imaginary part of $\operatorname{den}\left(F_{O L}\right)$.

\begin{tabular}{|l|c|c|c|c|}
\hline stable ? & $\tau$ & $\alpha$ & Nyquist & time response \\
\hline yes & $T_{e}$ & 1 & fig 2.1 & fig 3.1 \\
yes & $T_{e}$ & 10 & fig 2.2 & fig 3.2 \\
no & $5 T_{e}$ & 1 & fig 4.1 & fig 5.1 \\
yes & $5 T_{e}$ & 3 & fig 4.2 & fig 5.2 \\
no & $10 T_{e}$ & 3 & fig 6.1 & fig 7.1 \\
no & $10 T_{e}$ & 20 & fig 6.2 & fig 7.2 \\
\hline
\end{tabular}

The cases given in the array are shown with some simulations to highlight the influence on the stability of the parameter $\alpha$ with respect to the delay $\tau$.

On figure 2, we can see the results with different values for $\alpha$.
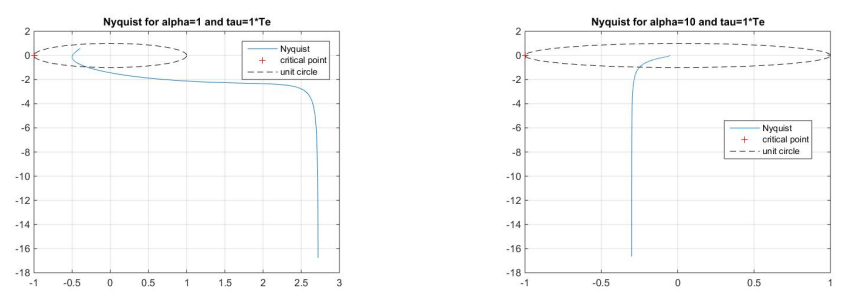

Figure 2. Results for $\tau=T_{e}, \alpha=1$ and $\alpha=10$.
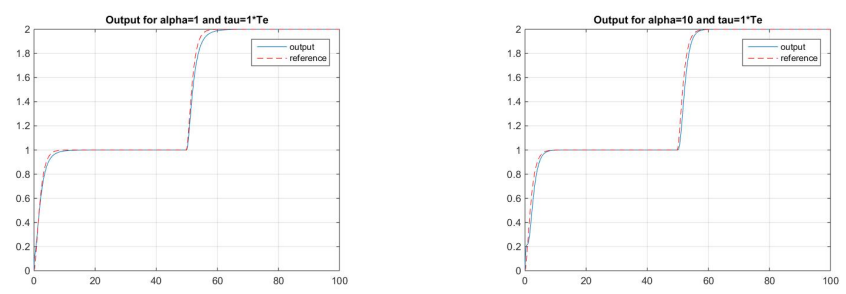

Figure 3. Output for $\tau=T_{e}, \alpha=1$ and $\alpha=10$.

On the first one, with $\alpha=1$, the critical point $(-1,0)$ is outside 
the Nyquist diagram. It means that the system remains stable. It is also confirmed on the first plot of the figure 3 .

On the second plot of figure 2, for $\alpha=10$, we can observe that the system remains stable and the Nyquist diagram is centered around the origin. It means that the system is more robust in this case because of the gain margin. But it is not clear with this academical example.

Then, the same simulations are made with a delay $\tau=5 T_{e}$. We can see on the first Nyquist diagram of the figure 4 that the critical point is encircled more than zero time. So it means that the system becomes unstable, this is confirmed by the first plot of the figure 5. When $\alpha$ is higher, we have seen before its effect. Indeed, the Nyquist diagram is tightened on the center and in this case the critical point is outside the closed outline. So it means that the system is stable and is confirmed with the second plot of the figure 5. On the first plot of figure 6 , we can
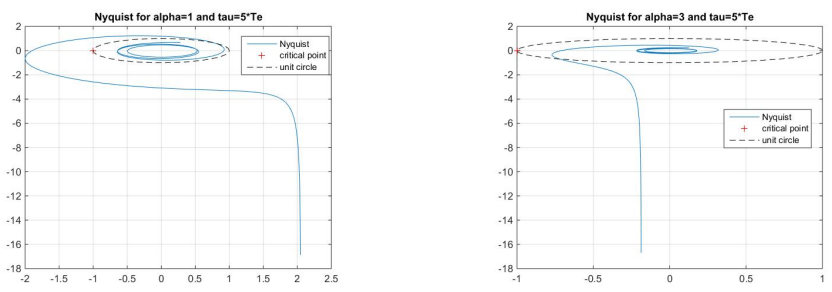

Figure 4. Results for $\tau=5 T_{e}, \alpha=1$ and $\alpha=3$.
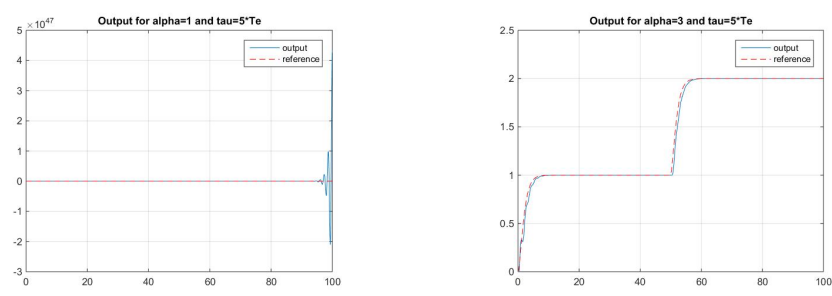

Figure 5. Output for $\tau=5 T_{e}, \alpha=1$ and $\alpha=3$.

see the Nyquist diagram for $\alpha=3$ and $\tau=10 T_{e}$. We observe that the critical point is encircled and so the system is unstable, it is confirmed with the first plot of figure 7 . With the second plot of figure 6 , we can see that when the parameter $\alpha$ goes up, the system is at the limit of the stability but it stills unstable, as we can see on the second plot of figure 7 . It means that when the delay is too high, it is difficult to stabilize the system however simple it is. More, we can see on figure 6.2 that when the Nyquist diagram is near the critical point, it induce some swings. It is confirmed by the second plot of the figure 7 .

\section{B. Second order system}

In this subsection, the same method will be applied on a second order unknown delayed system, but just one case. In a first time we give the $F_{C L}$ and $F_{O L}$, then the value of the denominator in function of $x$ and $y$. We consider the following system:

$$
\frac{Y(s)}{U(s)}=\frac{e^{-\tau s}}{s^{2}+1.4 s+1}
$$
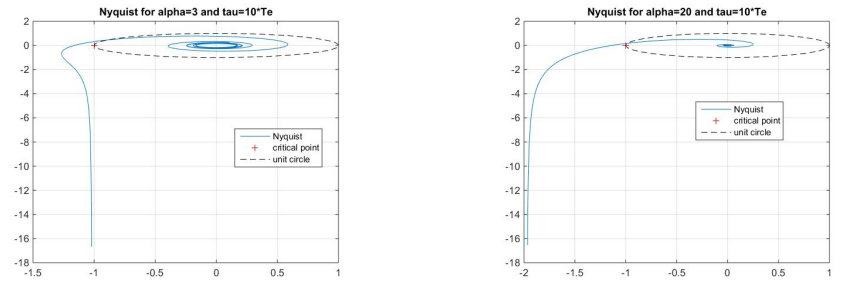

Figure 6. Results for $\tau=10 T_{e}, \alpha=3$ and $\alpha=20$.
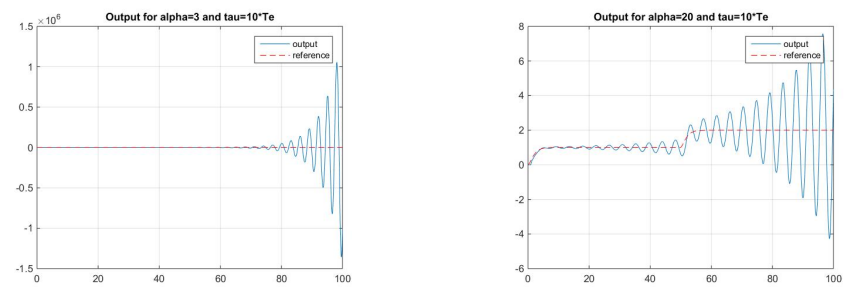

Figure 7. Output for $\tau=10 T_{e}, \alpha=3$ and $\alpha=20$.

which is controlled with MFC and $C(s)=K_{p}=0.05$. In a first time we have to obtain the closed-loop and the open-loop transfer function. The closed-loop transfer function is:

$$
\frac{Y(s)}{Y_{r e f}(s)}=\frac{T_{e}\left(s+\alpha K_{p}\right)}{e^{\tau s} T_{e} \alpha\left(s^{2}+1.4 s+1\right)\left(1-e^{-T_{e} s}\right)+A(s)}
$$

Using (9), the open-loop transfer function is defined such as:

$$
F_{O L}(s)=\frac{T_{e}\left(s+\alpha K_{p}\right)}{e^{\tau s} T_{e} \alpha\left(s^{2}+1.4 s+1\right)\left(1-e^{-T_{e} s}\right)+A(s)-B(s)}
$$

With $A(s)=\left(1-e^{-T_{e} s}+T_{e} \alpha K_{p}\right)$ and $B(s)=T_{e}\left(s+\alpha K_{p}\right)$. For the number of unstable zeros, it is the same, we find $z=$ $-\alpha K_{p}$. For the poles the calcul of the denominator gives:

$$
\begin{gathered}
\operatorname{Im}(\operatorname{den}(x, y))=T_{e} \alpha e^{\tau x} \sin (\tau y)\left(x^{2}-y^{2}+1.4 x+1\right) \\
+T_{e} \alpha e^{x \tau} \cos (\tau y)(2 x y+1.4 y) \\
-e^{-T_{e} x+\tau x} T_{e} \alpha \cos \left(-T_{e} y\right) \cos (\tau y)(2 x y+1.4 y) \\
-T_{e} \alpha e^{-T_{e} x+\tau x} \cos \left(-T_{e} y\right) \sin (\tau y)\left(x^{2}-y^{2}+1.4 x+1\right) \\
-e^{-T_{e} x+\tau x} \sin \left(-T_{e} y\right) \cos (\tau y)\left(x^{2}-y^{2}+1.4 x+1\right) T_{e} \alpha \\
-e^{-T_{e} x+\tau x} T_{e} \alpha \sin (\tau y) \sin \left(-T_{e} y\right)(2 x y+1.4 y) \\
-e^{-T_{e} x} \sin \left(-T_{e} y\right)-T_{e} y
\end{gathered}
$$

And for the real part:

$$
\begin{gathered}
\operatorname{Re}(\operatorname{den}(x, y))=T_{e} \alpha e^{\tau x} \cos (\tau y)\left(x^{2}-y^{2}+1.4 x+1\right) \\
+T_{e} \alpha e^{\tau x} \sin (\tau y)(-2 x y-1.4 y) \\
-e^{-T_{e} x} \cos \left(-T_{e} y\right) T_{e} \alpha e^{\tau x} \cos (\tau y)\left(x^{2}-y^{2}+1.4 x+1\right) \\
+e^{-T_{e} x+\tau x} T_{e} \alpha \cos \left(-T_{e} y\right) \sin (\tau y)(2 x y+1.4 y) \\
+e^{-T_{e} x+\tau x} T_{e} \alpha \sin \left(-T_{e} y\right) \cos (\tau y)(2 x y+1.4 y) \\
+e^{-T_{e} x+\tau x} T_{e} \alpha \sin (\tau y) \sin \left(-T_{e} y\right)\left(x^{2}-y^{2}+1.4 x+1\right)+1 \\
-e^{-T_{e} x} \cos \left(-T_{e} y\right)-T_{e} x
\end{gathered}
$$


Similarly to the first order case, we just plot a $3 d$ plot because the results remain the same whatever the values of the parameters. On figure 8 , the first plot shows that we need to focus on two zones: $x \in[0.5 ; 1]$ and $y \in[-1 ;-0.5]$ and around the origin. Both cases are presented in the second and the third plot of the figure 8 . So we can conclude that there is no unstable pole, it means that the Nyquist diagram has to make zero encirclement around the critical point. For the
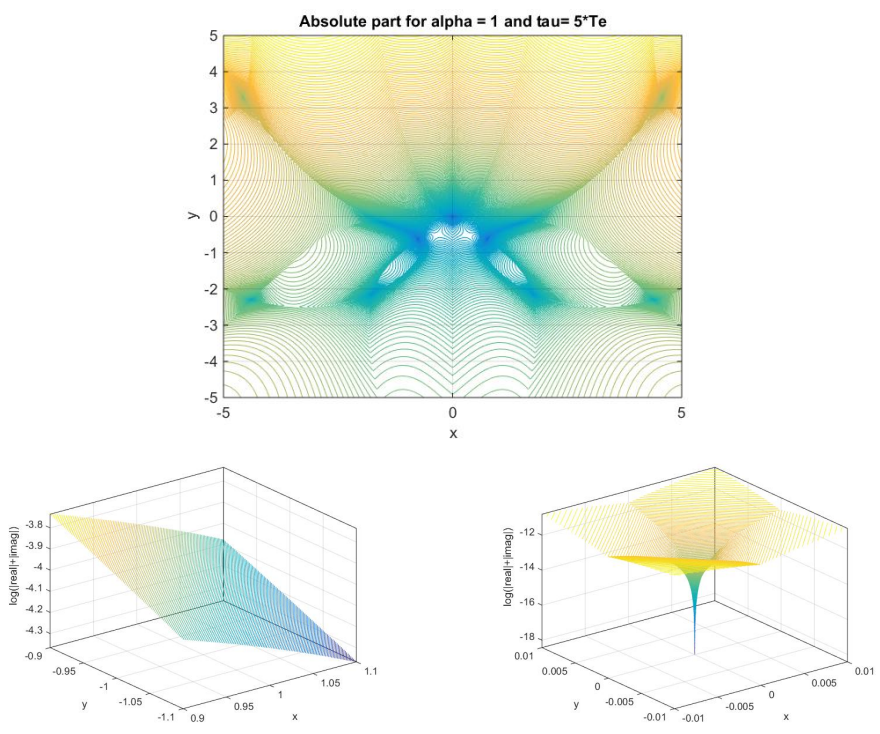

Figure 8. Logarithm of the sum of the absolute value of the real and imaginary part of the denominator.

following, we use the same presentation, i.e. an array contains a summary of the results and the simulations show them.

\begin{tabular}{|l|c|c|c|c|}
\hline stable ? & $\tau$ & $\alpha$ & Nyquist & time response \\
\hline no & $5 T_{e}$ & 1 & fig 9.1 & fig 10.1 \\
yes & $5 T_{e}$ & 5 & fig 9.2 & fig 10.2 \\
yes & $5 T_{e}$ & 10 & fig 9.3 & fig 10.3 \\
\hline
\end{tabular}

In this general section, using the Nyquist diagrams and applying the generalized Nyquist stability criterion a frequential analysis for the stability analysis was proposed. It was strengthened with some simulations in different cases. We show the influence of the parameter $\alpha$ on the stability. Indeed, the tunning of this parameter can make the system unstable.

It is important to note that to manage this analysis, the model of the system need to be well-known. Indeed, according this, all the equations and the different transfer functions can be written.

\section{CONCLUSION}

The problem of the stability of unknown delayed systems has been treated here in a model-free control context. A discussion about the choice of a parameter was made and the influence of this parameter on the stability regarding to the delay has been shown. Thanks to the generalized Nyquist stability criterion, a frequential analysis was made to study the stability. But
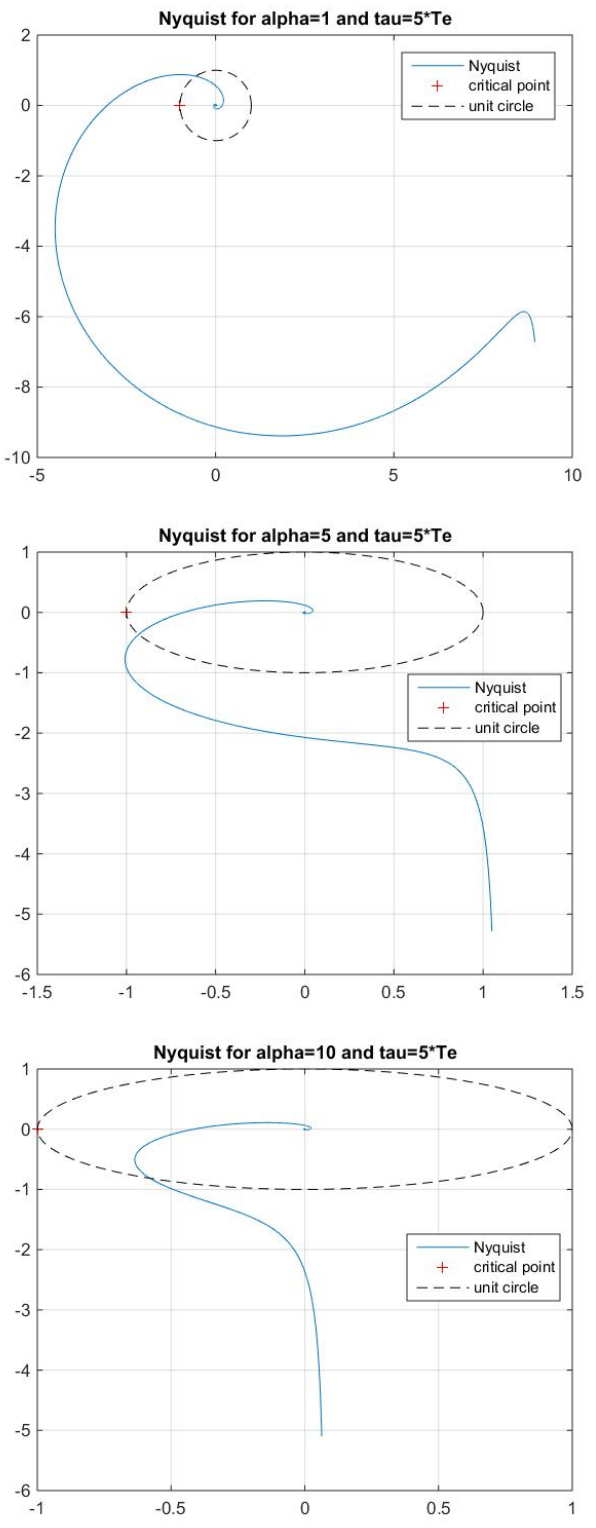

Figure 9. Nyquist for $\tau=5 T_{e}, \alpha=1, \alpha=5$ and $\alpha=10$.

some limits appear. Indeed, to manage this analysis, the model of the system has to be well known as well as the delay. More, sometimes the calculation to obtain some solutions are complex.

In a future work, the frequential analysis proposed in this paper will be used to analyse the performance of the system in closed-loop and to tune the $\alpha$ parameter as well as needed.

\section{REFERENCES}

[1] M.Fliess and C.Join. Model-free control, International Journal of Control, 86(12), 2228-2252, 2013

[2] C.Join, F.Chaxel and M.Fliess. Intelligent controllers on cheap and small programmable devices, 2nd Int. Conf. Control FaultTolerant Syst., Nice, 2013 

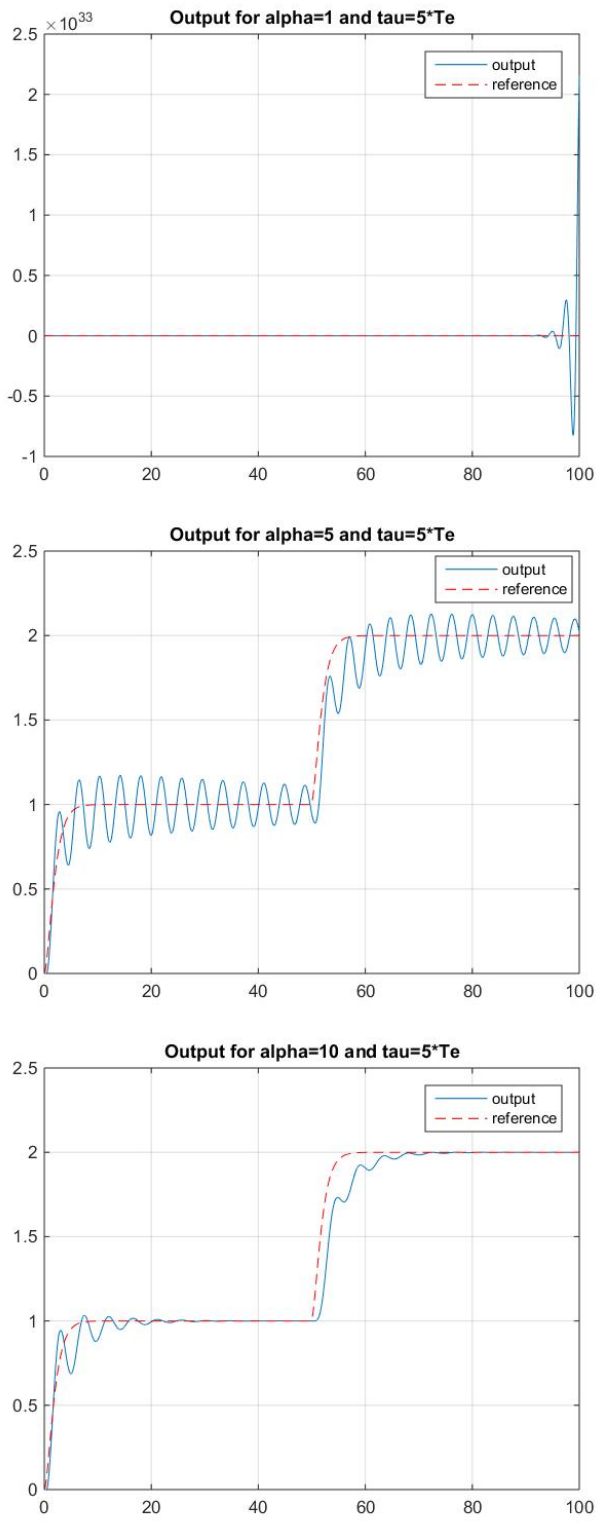

Figure 10. Outputs for $\tau=5 T_{e}, \alpha=1, \alpha=5$ and $\alpha=10$.
[3] P.A.Gédouin, E.Delaleau, J.M.Bourgeot, C.Join, S.Arab-Chirani and S.Calloch. Experimental comparison of classical pid and model-free control : Position control of a shape memory alloy active spring. Control Engineering Practice, 2011, 19, 433-441

[4] M.Doublet, C.Join and F.Hamelin. Model-free control for unknown delayed systems, SysTol'16: 3rd International Conference on Control and Fault-Tolerant Systems, 2016

[5] F.J.Carrillo and F.Rotella. Some contributions to estimation for model-free control, 17th IFAC Symposium on System Identification SYSID 2015, Beijing, China, 2015

[6] C.A.Desoer and Y-T.Wang. On the Generalized Nyquist Stability Criterion IEEE Transactions on Automatic Control, vol. ac-25, no, 1980

[7] B.Wen, D.Boroyevich, P.Mattavelli, Z.Shen and R.Burgos. Experimental verification of the Generalized Nyquist stability criterion for balanced three-phase ac systems in the presence of constant power loads Energy Conversion Congress and Exposition (ECCE), 2012 IEEE, 2012

[8] F.Lafont, J-F.Balmat, N.Pessel and M.Fliess. A model-free control strategy for an experimental greenhouse with an application to fault accommodation, Comput. Electron. Agricult., 2015, p. 139-149.

[9] J.G.Ziegler and N.B.Nichols. Optimum Settings for Automatic Controllers , 1942.

[10] K.J.Astrom, C.C. Hang and B.C. Lim. A new Smith predictor for controlling a process with an integrator and long dead-time. IEEE transactions on automatic control, 39(2), 343-345, 1994.

[11] M.R.Garcia, C.Vilas, L.O.Santos and A.A.Alonso. A Robust Multi-Model Predictive Controller for Distributed Parameter Systems. Journal of Process Control, 22, 60-71, 2012.

[12] R.Luebbers, F.Hunsberger, K.S.Kunz, R.Standler and M.Schneider. A frequency-dependent finite-difference timedomain formulation for dispersive materials. Electromagnetic Compatibility, IEEE Transactions on. 32 (3): 222-227, 1990.

[13] S.Majhi and D.P.Atherton. Obtaining controller parameters for a new smith predictor using autotuning. Automatica, 36, 1651$1658,2000$. 